\title{
Cell-Specific Ecdysone-Inducible Expression of FLP Recombinase in Mammalian Cells
}

BioTechniques 25:868-875 (November 1998)

\author{
Janet A. Sawicki, Bob Monks \\ and Rebecca J. Morris \\ Lankenau Medical Research \\ Center, Wynnewood, PA, USA
}

\section{INTRODUCTION}

The ability to manipulate genomes of higher organisms using site-directed recombinases such as FLP, from Saccharomyces cerevisiae, and Cre, from bacteriophage $\mathrm{P} 1$, is widely recognized as an important way to explore the molecular basis for developmental processes. The simplicity by which these recombinases work to catalyze excisions, integrations, inversions or translocations at specific target sites in DNA also suggests that with the development of strategies to regulate the recombination events they mediate, these enzymes will be important tools for introducing specific genetic alterations required by gene therapy.

Several studies have shown that recombinase-mediated DNA alterations can be regulated either (i) by controlling the expression of the recombinase with inducible promoters $(3,5-7,11)$, (ii) by introducing recombinase mRNA or protein into cells $(1,8)$ or $(i i i)$ by regulating the activity of an expressed recombinase (9). When applied to whole animals, however, each of these approaches is either impractical or has the disadvantage that cell-specific regulation cannot be attained.

Recently, the insect steroid hormone ecdysone has been shown to be an efficient and potent inducer of gene expression in cultured cells and transgenic mice (12). In this report, we place the FLP coding sequence under the regulation of an ecdysone-responsive promoter. In addition, by using a cell-specific promoter to regulate the expression of elements required for activation of the ecdysone-responsive promoter, we temporally induce FLP-mediated, site-specific recombination events in specific cells and their descendants.

\section{MATERIALS AND METHODS}

\section{Plasmid Constructs}

pNEOßGAL, pFRT $\beta G A L$ and pOG44 were purchased from Stratagene (La Jolla, CA, USA). The pVgRXR and pIND/lacZ plasmids are components of the Ecdysone-Inducible Mammalian Expression System (Invitrogen, Carlsbad, CA, USA). More information on these plasmids is available on the Invitrogen Web site (http://www. invitrogen.com). The $\mathrm{pIND} / F L P$ plasmid, which contains the ecdysone responsive minimal heat shock promoter (IND), was constructed by ligating the 2030-bp XbaI/ApaI fragment containing the FLP sequence of pOG44 into the $X b a \mathrm{I}$ and ApaI sites in the multiple cloning site of pIND. pK19/VgRXR was constructed in two steps. First, three fragments were ligated into a HindIII/PstI-digested pUC19 vector sequence as follows: (i) a PstI/ApaLI fragment containing the VgEcR and TKpA sequences of pVgRXR, (ii) a RXRBGHpA sequence amplified from $\mathrm{pV}$ gRXR using two oligonucleotides; 5'CCATCGATATATCACCATGGACAC C- $3^{\prime}$ and $5^{\prime}$-TCGTAAGCTTCCGTCTATCAGGGCGA-3' (underlined nucleotides correspond to ClaI and HindIII sites) and (iii) a murine K19 promoter sequence (10) amplified from 
a plasmid pK19ßgal using two oligonucleotides; 5'-AGTTGTGCACGTTGGCAGTAGCTTT-3' and 5'-CCATCGATATGATGAGGAGGGAGACC-3' (underlined nucleotides correspond to ApaLI and ClaI sites). The resulting 8070-bp plasmid was digested with PstI and NdeI. To introduce a second K19 promoter sequence into the construct $5^{\prime}$ to the $\mathrm{VgEcR}$ sequence, the resulting 7815-bp fragment was ligated to another fragment containing the same K19 promoter sequence as above, this time amplified from pK19ßgal using two different oligonucleotides, 5'-AGTTCATATGGTTGGCAGTAGCTTT-3' and 5'-TCGTCTGCAGATGATGAGGAGGGAGACC-3' (underlined nucleotides correspond to NdeI and PstI sites).

\section{Cell Culture and Transfection}

NIH 3T3 cells $\left(7.5 \times 10^{6}\right)$ (Accession No. CRL-1658; ATCC, Rockville, $\mathrm{MD}$, USA) were electroporated in phosphate-buffered saline (PBS), $\mathrm{pH}$ 7.3, using a Gene Pulser ${ }^{\circledR}(960 \mu \mathrm{F}, 0.3$ $\mathrm{kV}, 0.4-\mathrm{cm}$ electrode) (Bio-Rad, Hercules, CA, USA). Ten micrograms of each plasmid DNA were used for each electroporation, irrespective of the number of plasmids that were co-transfected. After electroporation, $8 \%$ of the cells were seeded into a Falcon ${ }^{\circledR}$ Tissue Culture Treated Glass Culture Slide (Becton Dickinson Labware, Bedford, MA, USA); the balance of the cells were seeded equally into three $100-x$ 15-mm tissue culture dishes. Twentyfour hours after electroporation, the culture medium was replaced with fresh culture medium or medium containing muristerone A (Invitrogen, Carlsbad, CA, USA). After an additional $24 \mathrm{~h}$ in culture, cells in dishes were fixed for $5 \mathrm{~min}$ at room temperature in $2 \%$ gluteraldehyde and histochemically stained at $37^{\circ} \mathrm{C}$ for $\beta$-galactosidase $(\beta$ gal) activity. Stained cells were viewed with a Model M3Z Zoom Stereomicroscope (Wild Leitz, Malvern, PA, USA) and counted. Fluorescing cells in the glass slide chambers were detected and photographed using a Axioskop ${ }^{\circledR} 20$ Fluorescent Microscope (Carl Zeiss, Thornwood, NY, USA) equipped with a fluorescein isothiocyanate (FITC) filter $(488 \mathrm{~nm})$.

R1 embryonic stem (ES) cells were maintained at $37^{\circ} \mathrm{C}$ in $5 \% \mathrm{CO}_{2}$, balance air, in Dulbecco's modified Eagle medium (DMEM) supplemented with 10\% fetal bovine serum (Hyclone Laboratories, Logan, UT, USA), $\beta$-mercaptoethanol, nonessential amino acids, nucleoside stock (8) and with Recombinant Murine Leukemia Inhibitory Factor (LIF) (106 U/mL) (Life Technologies, Gaithersburg, MD, USA). Before a transfection experiment, cells were cultured in bacteriological culture dishes (Becton Dickinson Labware) in medium lacking LIF for 4 days. The simple embryoid bodies that formed were seeded into tissue culture dishes. After 2 days of culture, adhering embryoid bodies and cell outgrowths were harvested and electroporated in PBS, $\mathrm{pH} 7.3$, using a Gene Pulser $(500 \mu \mathrm{F}$, $0.3 \mathrm{kV}, 0.4-\mathrm{cm}$ electrode). Ten micrograms of each plasmid DNA were used. Electroporated cells were seeded into glass culture slides and treated with muristerone A for $24 \mathrm{~h}$. Following fixation with acetone for $10 \mathrm{~min}$ at $-20^{\circ} \mathrm{C}$, cells were incubated overnight at $4^{\circ} \mathrm{C}$ in a humidified chamber with a monoclonal antibody to K19 (1:10) (Catalog No. RPN1165; Amersham Pharmacia Biotech, Piscataway, NJ, USA) and a rabbit polyclonal antibody to $\beta$-gal $(1: 100)$ (Catalog No. 7-063100; 5 Prime $\rightarrow 3$ Prime, Boulder, CO, USA). The secondary antibodies used were a CY3conjugated goat anti-mouse $\mathrm{IgG}$ and a FITC-conjugated goat anti-rabbit IgG (Jackson ImmunoResearch, West Grove, PA, USA).

\section{RESULTS}

Figure 1 shows the DNA constructs used in this study. In the plasmid pNEO $\beta$ GAL, the $\beta$-gal coding sequence has been disrupted by a neo gene that is flanked by FLP recognition target (FRT) sites in direct orientation. The FRT-flanked neo casette is inserted after the first codon (AUG) of the $\beta$-gal coding sequence; therefore, not even a truncated $\beta$-gal protein is made from the disrupted $\beta$-gal sequence. FLP-

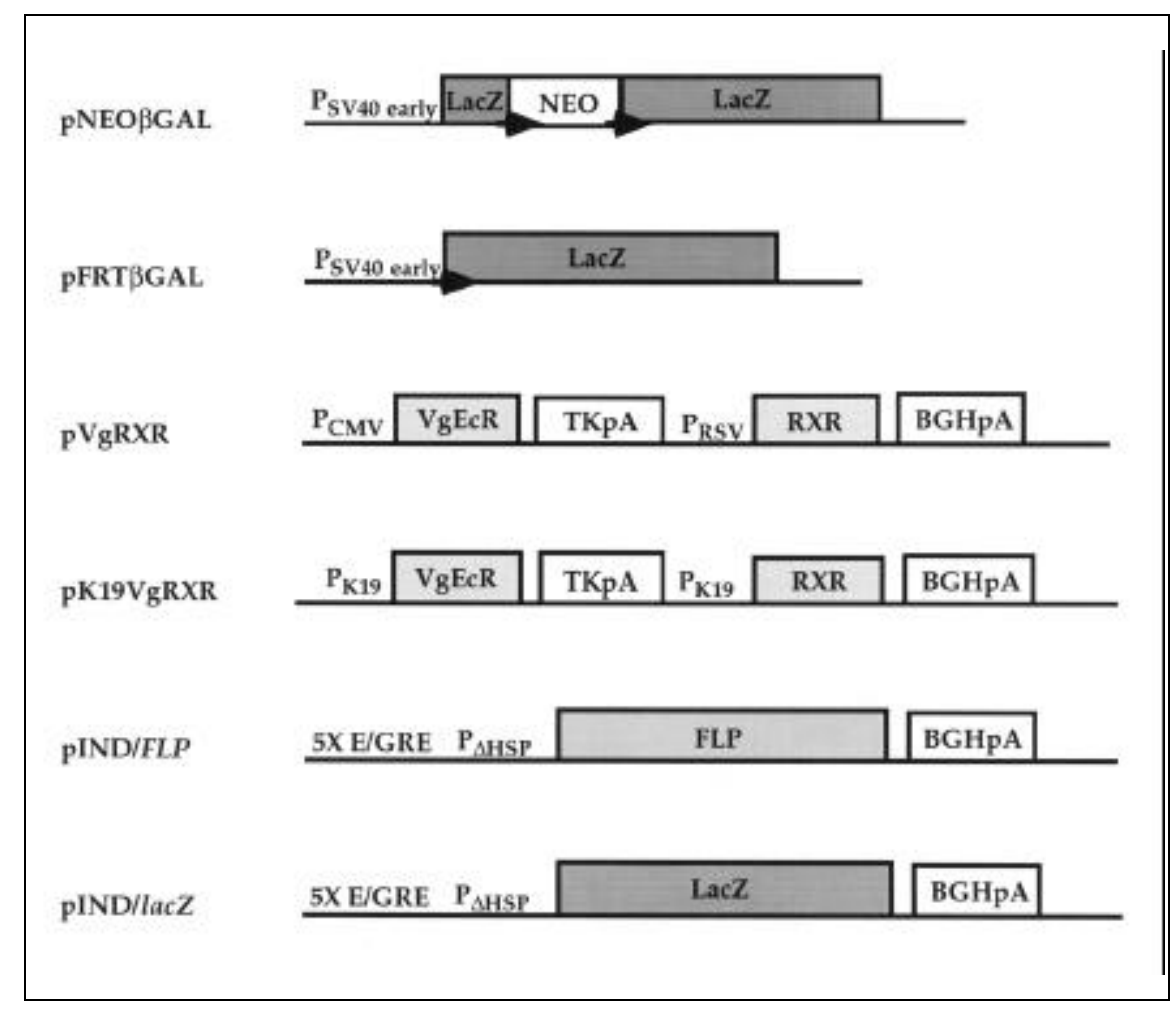

Figure 1. Plasmid constructs. $P_{\mathrm{SV} 40}$ early, early promoter of SV40; NEO, neomycin-resistance gene; $\mathrm{P}_{\mathrm{CMV}}$, cytomegalovirus immediate early promoter; $\mathrm{VgEcR}$, modified ecdysone receptor; TKpA, thymidylate kinase polyadenylation site; $\mathrm{P}_{\mathrm{RSV}}$, Rous sarcoma virus promoter; RXR, retinoid-X receptor; BGHpA, bovine growth hormone polyadenylation signal; E/GRE, modified ecdysone response element; $\mathrm{P}_{\Delta \mathrm{HSP}}$, minimal heat shock promoter. FRT sequences are indicated by the black arrow heads. 


\section{Research Reports}

mediated recombination between these FRTs results in the excision of neo, thereby reconstituting an intact and functional $\beta$-gal sequence. The plasmid pFRT $\beta$ GAL contains an intact $\beta$-gal coding sequence preceded by a single FRT. Elements required for ecdysone induction are housed in the plasmids $\mathrm{pVgRXR}, \mathrm{pIND} / F L P$ and $\mathrm{pIND} / \mathrm{lacZ}$. pVgRXR contains genes encoding a heterodimer of the ecdysone receptor (VgEcR; derived from Drosophila and modified to contain the VP16 transactivation domain) and the retinoid-X receptor (RXR; derived from mammalian cells). This heterodimer binds to a modified ecdysone response element (E/GRE) in the presence of ecdysone (or a natural analog, muristerone A). In $\mathrm{pIND} / F L P$, the FLP recombinase sequence has been placed under the control of the inducible promoter sequence, IND, containing the modified E/GRE linked to a minimal heat shock promoter. The same promoter controls activation of the $\beta$-gal encoding lac $Z$ gene in pIND/lacZ.

Figure 2 outlines the method for combining the genetic elements required for ecdysone-induction with those required for FLP-mediated recombination, which can introduce site-

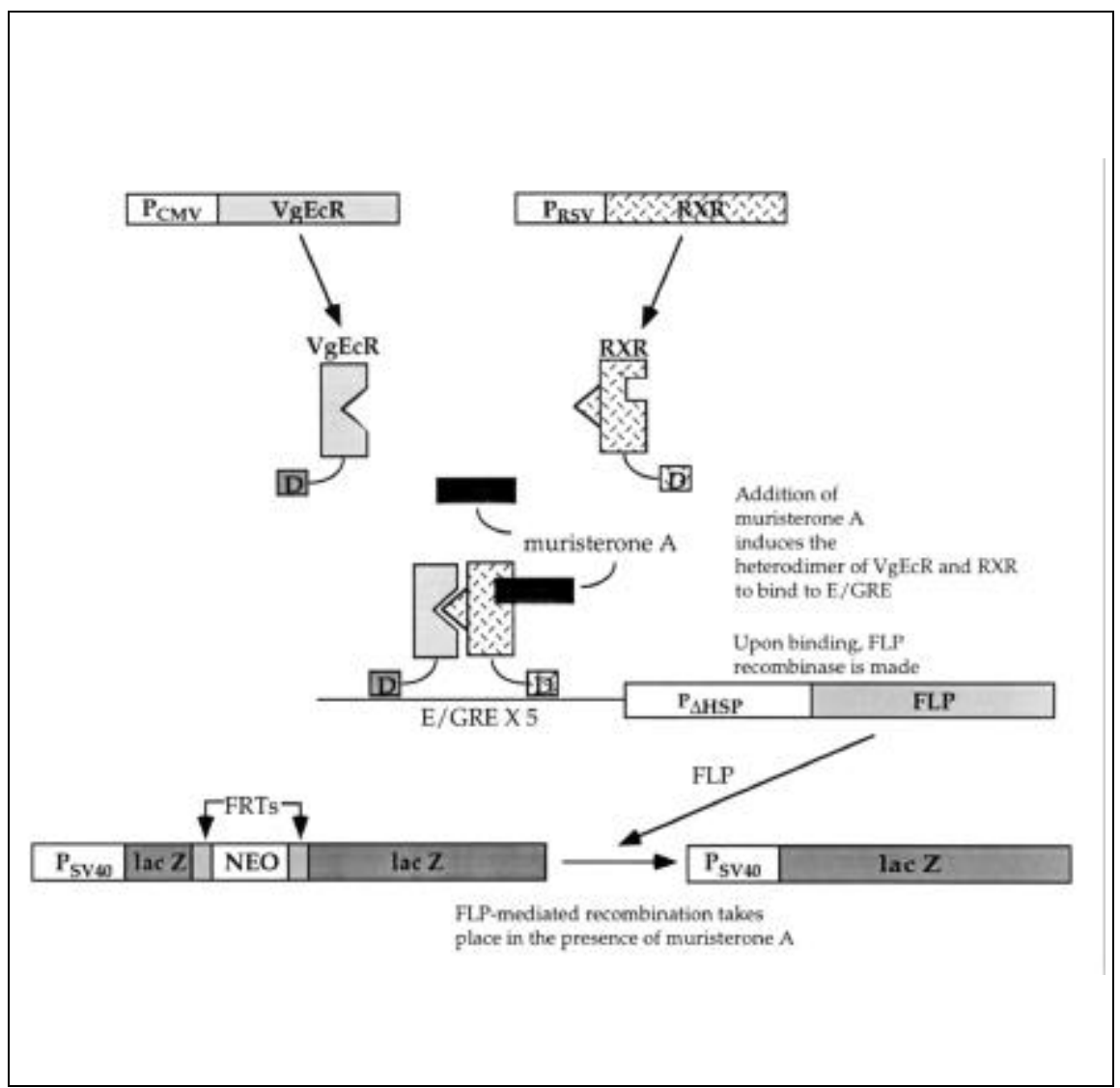

Figure 2. Elements required for ecdysone-induction of FLP-mediated recombination. Muristerone A is a natural analog of ecdysone. D indicates DNA-binding domain. Other elements are as described in Figure 1.

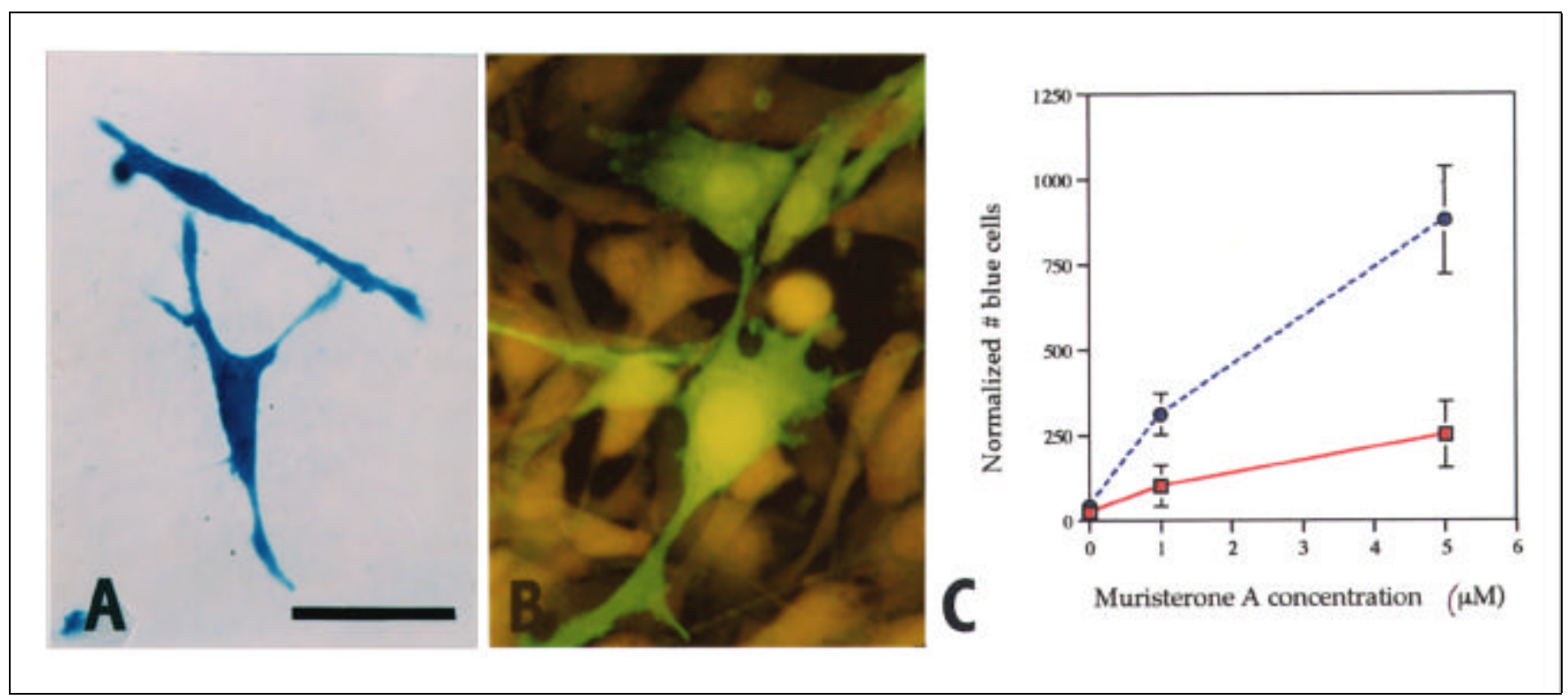

Figure 3. Transfection of NIH-3T3 cells to test for ability of muristerone A to induce FLP-mediated recombination. Cells in A and B were co-transfected with $\mathrm{pVgRXR}$, pIND/FLP, pNEOßGAL and $\mathrm{pCX}$-EGFP. They were then cultured in the presence of 0,1 or $5 \mu \mathrm{M}$ muristerone A. (A) Blue-staining cells, indicating cells expressing $\beta$-gal activity. The bar represents $10 \mu \mathrm{M}$. (B) Green GFP-expressing cells. Non-expressing cells appear orange, the result of counterstaining with propidium iodide. (C) Dosage-dependent muristerone A induction of FLP-mediated recombination. The red line corresponds to cells co-transfected with $\mathrm{pVgRXR}$, pIND/FLP and pNEOßGAL; the blue line corresponds to cells co-transfected with $\mathrm{pVgRXR}$ and pIND/lacZ. The number of $\beta$-gal expressing cells was normalized to the number of GFP-expressing cells. 
specific changes in DNA in an inducible manner. When cells that express the ecdysone receptor-RXR heterodimer are treated with muristerone, FLP recombinase under the control of the IND promoter will be expressed. Site-directed, FLP-mediated recombination events will then occur between DNA sequences containing FRTs. Figure 2 shows a FLP-mediated recombination event that results in the excision of a neo sequence, thereby reconstituting an intact $l a c \mathrm{Z}$ gene.

To test whether FLP-mediated recombination can actually be induced by ecdysone, NIH-3T3 cells were transiently co-transfected with the plasmids pVgRXR, pIND/FLP and pneoßgal. Twenty-four hours after electroporation, muristerone A was added to the culture medium at various concentrations. After another $24 \mathrm{~h}$, cells were fixed and histochemically stained for $\beta$ gal activity (cells having $\beta$-gal activity stain blue; Figure 3A).

Several control transfections (C1C5) were also done. Table 1 summarizes the number of blue cells in each transfection, with and without muristerone A induction. For each transfection, in addition to the plasmids indicated in Table I, cells were co-transfected with a plasmid, pCX/EGFP, encoding a variant green fluorescent protein (GFP). The number of blue cells was normalized to the number of cells expressing GFP (Figure 3B). Our ability to transfect cells at a reasonable rate is demonstrated by the high number of cells that stain blue in $\mathrm{C} 1$, i.e., cells transfected with pFRT $\beta$ GAL. No blue cells were observed in cells transfected with pIND/ lac Z (C2), either in the absence or presence of muristerone $\mathrm{A}$, indicating that IND is not leaky and is not activated in the absence of the VgEcR-RXR heterodimer. In cells transfected with $\mathrm{pV}$ gRXR and pIND/lacZ (C3), the presence of a small number of blue cells in the absence of muristerone indicates that, in the presence of the VgEcR-RXR heterodimer, the IND promoter is slightly leaky. Activation of this promoter is shown to be responsive to muristerone in a dose-dependent manner, as the number of blue cells increased with increased concentrations of muristerone (also see Figure 3C). No blue cells were observed when cells were transfected with pNEOBGAL $(\mathrm{C} 4)$ indicating that there is little, if any, spontaneous excision of the FRT-flanked neo sequence. The absence of blue cells when cells were electroporated with PBS lacking DNA (C5) confirmed the specificity of the histochemical stain for lacZ-encoded $\beta$-gal activity.

When cells were transfected with pVgRXR, pIND/FLP and pNEO $\beta$ GAL, very few cells stained blue in the absence of muristerone. The number of blue cells increased significantly in a dose-dependent manner when cells were treated with muristerone (Figure $3 \mathrm{C})$. In the absence or presence of muristerone, the number of blue cells is lower than in $\mathrm{C} 3$, probably reflecting the requirement of two events for $\beta$-gal activity; namely induction of FLP and FLP-mediated excision of neo. These observations, together with the results of the control transfections, lead us to conclude that FLP-mediated, site-specific recombination events can be tightly regulated by the induced activation of an ecdysone-responsive promoter.

As mentioned, the expression of FLP recombinase or the activation of its enzyme activity has previously been successfully placed under the regulation of other inducible promoters. Using cell-specific promoters, its expression has also been activated in specific cells (4). In principle, the two-tiered regulatory system outlined here has the added advantage that judicious selection of a promoter to regulate the expression of the $\mathrm{VgEcR}$ and RXR elements should allow temporal induction of FLP-mediated changes in DNA in specific cells in response to muristerone A. To test whether this is in fact the case, we transfected ES with pK19VgRXR, pIND/FLP and pNEO$\beta G A L$. In $\mathrm{pK} 19 \mathrm{VgRXR}$, the expression of $\mathrm{VgEcR}$ and RXR is regulated by the promoter of the murine keratin 19 (K19) gene. K19 is known to be expressed upon the differentiation of ES cells into organized structures known as embryoid bodies (13). Following transfection, ES cells were grown under conditions that induce differentiation and treated with muristerone A. Twentyfour hours later, fixed cells were immunostained for both $\beta$-gal and K19. $\beta$ gal protein, the consequence of FLP recombinase mediated reconstitution of 


\section{Research Reports}

Table I. Number of Cells Expressing $\beta$-gal Activity after Transfectiona

\begin{tabular}{|c|c|c|c|}
\hline \multirow[b]{2}{*}{ Plasmid DNA(s) } & \multicolumn{3}{|c|}{ Concentration of muristerone A } \\
\hline & none & $1 \mu \mathrm{M}$ & $5 \mu \mathrm{M}$ \\
\hline $\mathrm{pVgRXR}+\mathrm{pIND} / \mathrm{FLP}+\mathrm{pNEO} \beta \mathrm{GAL}$ & $24 \pm 6^{b}$ & $102 \pm 60$ & $252 \pm 98$ \\
\hline pFRT $\beta$ GAL (Control 1) & $3481 \pm 905$ & $3786 \pm 1563$ & $4644 \pm 1389$ \\
\hline pIND/LacZ (Control 2) & 0 & 0 & 0 \\
\hline pVgRXR + pIND/LacZ (Control 3) & $42 \pm 11$ & $313 \pm 6$ & $880 \pm 157$ \\
\hline pNEOßGAL (Control 4) & 0 & 0 & 0 \\
\hline no DNA (Control 5) & 0 & 0 & 0 \\
\hline
\end{tabular}

a functional lac Z sequence, was only observed in cells that also expressed K19 (Figure 4). $\beta$-gal protein was not observed in ES cells that were not treated with muristerone A.

\section{DISCUSSION}

These studies demonstrate that by combining the genetic elements required for ecdysone induction and for FLP recombination, it is possible to temporally change gene expression in specific cells. This system potentially has several practical applications in cultured cells, transgenic animals (excluding insects) and possibly in transgenic plants as well. Similar to a simpler system in which the sequence encoding FLP recombinase is placed under the control of a cell-specific promoter, the system described here is useful for the analysis of cell lineages in developing systems both in vitro and in vivo. However, the ecdysone/FLP system has the added advantage that if a given promoter is active in specific cells throughout development, the starting point at which one begins to track a cell lineage can be regulated by the administration of ecdysone. This added capability increases the potential for studying the progeny of specific cells in tissues that are in advanced stages of differentiation as well as in self-renewing tissues in the adult organism. The conditional activation of genes that is possible using this system facilitates the study of transgenes whose expression during embryogenesis is lethal, e.g., overexpressed oncogenes or dominant negatives. Using this system, it should also be possible to identify the cells from which primary tumors arise and to track metastatic foci deriving from a primary tumor. The results of

these experiments also suggest applications of this system to gene therapy strategies that are designed either to activate functional gene sequences in specific cell lineages at specified times or to ablate cells of a specified lineage.

Spatio-temporal expression of Cre recombinase has recently been achieved using tamoxifen induction of a modified ligand-binding domain of the human es-

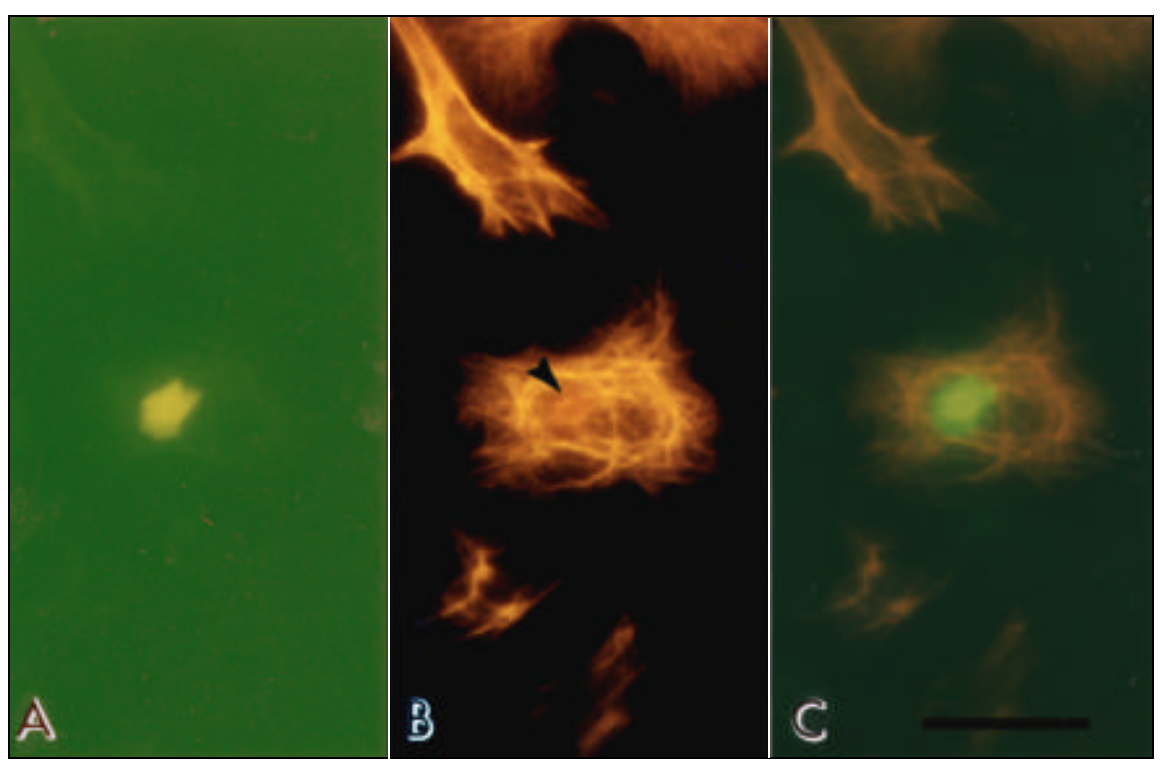

Figure 4. Cell-specific, ecdysone-induced FLP recombination in ES cells. Cells were co-transfected with $\mathrm{pK} 19 \mathrm{VgRXR}, \mathrm{pIND} / F L P$ and pNEOBGAL. After culturing in the presence of $5 \mu \mathrm{M}$ muristerone A, cells were doubly immunostained for $\beta$-gal and K19 using two different fluorescently labeled secondary antibodies. (A) A cell staining positive for $\beta$-gal, detected using an FITC filter. (B) The same field as in Panel A, but cells were viewed using a CY3 filter to detect K19 expressing cells. The $\beta$-gal-positive cell in Panel A is indicated with an arrow. (C) A double-exposure photograph of the same field taken with both the FITC and CY3 filters. The bar represents $10 \mu \mathrm{M}$. 
trogen receptor (2). Regulatory systems such as this one and the ecdysone-based system described here clearly empower the utility of site-specific recombinases.

\section{ACKNOWLEDGMENTS}

We thank M. Ikawa for the pCXEGFP plasmid; D. Telly for excellent technical assistance and C. Hobbs and T. O'Brien for critically reading the manuscript. This work was supported in part by NIH Grant No. CA45293 (R.J.M.).

\section{REFERENCES}

1.Baubonis, W. and B. Sauer. 1993. Genomic targeting with purified Cre recombinase. Nucleic Acids Res. 21:2025-2029.

2.Brocard, J., X. Warot, O. Wendling, N. Messaddeq, J.-L. Vonesch, P. Chambon and D. Metzger. 1997. Spatio-temporally controlled site-specific somatic mutagenesis in the mouse. Proc. Natl. Acad. Sci. USA
94:14559-14563.

3.Dang, D.T. and N. Perrimon. 1992. Use of a yeast site-specific recombinase to generate embryonic mosaics in Drosophila. Dev. Genet. 13:367-375.

4.Dymecki, S.M. 1996. Flp recombinase promotes site-specific DNA recombination in embryonic stem cells and transgenic mice. Proc. Natl. Acad. Sci. USA 93:6191-6196.

5.Golic, K.G. 1991. Site-specific recombination between homologous chromosomes in Drosophila. Science 252:958-961.

6.Golic, K.G. and S. Lindquist. 1989. The FLP recombinase of yeast catalyzes site-specific recombination in the Droposhila genome. Cell 59:499-509.

7.Kilby, N.J., M.R. Snaith and J.A.H. Murray. 1993. Site-specific recombinases: tools for genome engineering. Trends Genet. 9:413421 .

8.Konsolaki, M., M. Sanicola, T. Kozlova, V. Liu, B. Arca, C. Savakis, W.M. Gelbart and F.C. Kafatos. 1992. FLP-mediated intermolecular recombination in the cytoplasm of Drosophila embryos. New Biol. 4:551-557.

9.Logie, C. and A.F. Stewart. 1995. Ligandregulated site-specific recombination. Proc. Natl. Acad. Sci. USA 92:5940-5944.

10.Lussier, M., M. Filion, J.G. Compton, J.H.
Nadeau, L. Lapointe and A. Royal. 1990. The mouse keratin 19-encoding gene: sequence, structure and chromosomal assignment. Gene 95:203-213.

11.Morris, A.C., T.L. Schaub and A.A. James. 1991. FLP-mediated recombination in the vector mosquito, Aedes aegypti. Nucleic Acids Res. 19:5895-5900.

12.No, D., T. Yao and R.M. Evans. 1996. Ecdysone-inducible gene expression in mammalian cells and transgenic mice. Proc. Natl. Acad. Sci. USA 93:3346-3351.

13.Robertson, E.J. 1987. Embryo-derived stem cell lines, p. 71-112. In E.J. Robertson (Ed.), Teraotocarcinoma and Embryonic Stem Cells. A Practical Approach. IRL Press, Washington, D.C.

Received 12 March 1998; accepted 14 May 1998.

Address correspondence to:

Dr. Janet A. Sawicki

Lankenau Medical Research Center

100 Lancaster Avenue

Wynnewood, PA 19096, USA

Internet: sawicki@voicenet.com 